\title{
JACOBSON RADICAL OF FILTERED ALGEBRAS
}

\author{
ERAZM J. BEHR
}

\begin{abstract}
Using elementary graded ring theory methods we show that the Jacobson radical of certain filtered algebras is zero. We then use this to propose a simpler and more general proof of one of the main results contained in [1].
\end{abstract}

Throughout this note, $K$ will be a commutative ring with 1 . An algebra $A$ will mean a $K$-module equipped with an associative multiplication, whose identity element is $1 \in K$.

$A$ is a filtered algebra if there is an ascending chain of $K$-submodules of $A$, namely $(0)=A_{(-1)} \subseteq A_{(0)} \subseteq A_{(1)} \subseteq \cdots$, such that $A_{(i)} A_{(j)} \subseteq A_{(i+j)}$ for all nonnegative integers $i$ and $j$. We will consider only nontrivial filtrations, i.e. the case when $A \neq A_{(0)}$, and we will always assume that $1 \in A_{(0)}$. For each element $a$ of $A$ we define $d(a)$ to be the smallest integer $i$ for which $a \in A_{(i)}$.

A filtration of a $K$-algebra $A$ gives rise to a corresponding graded object, the associated graded algebra of $A$, defined as the $K$-direct sum $\operatorname{Gr}(A)=A_{(0)} / A_{(-1)} \oplus$ $A_{(1)} / A_{(0)} \oplus A_{(2)} / A_{(1)} \oplus \cdots$ in which multiplication is given by

$$
\left(a+A_{(i-1)}\right)\left(b+A_{(j-1)}\right):=a b+A_{(i+j-1)} \text { for any } i, j \geqslant 0
$$

with the above rule extended by linearity. For an element $a \neq 0$ of the filtered algebra $A$ we define $\operatorname{gr}(a)$ to be the element $a+A_{(d(a)-1)}$ of $\operatorname{Gr}(A)$. We also declare that $\operatorname{gr}(a)=0$ if and only if $a=0$.

$J(A)$ will denote the Jacobson radical of $A$.

THEOREM. Let $A$ be an algebra filtered in such a way that $\operatorname{Gr}(A)$ has no zero divisors. Then $J(A)=(0)$.

Proof. Take any $a \in J(A)$. There exists a nonzero $b \in A$ for which $(1-a) b=1$. Suppose that $d(a)>0$. Then $\operatorname{gr}(1-a)=\operatorname{gr}(a)$, and it follows that $\operatorname{gr}(a) \operatorname{gr}(b)=$ $\operatorname{gr}(1-a) \operatorname{gr}(b)=1+A_{(d(a)+d(b)-1)}=0$. Since both $\operatorname{gr}(a)$ and $\operatorname{gr}(b)$ are nonzero, we have a contradiction with the hypothesis. Hence $d(a) \leqslant 0$, meaning that $J(A) \subseteq$ $A_{(0)}$. Now let $b \in A$ with $d(b)>0$, and let $a$ be an element of $J(A)$ once again. Then $a b \in J(A) \subseteq A_{(0)}$, so that $\operatorname{gr}(a) \operatorname{gr}(b)=a b+A_{(d(a)+d(b)-1)}$ is zero (since $d(a)+d(b)-1 \geqslant 0)$. This implies that $a=0$, thus proving the assertion.

The above theorem allows us to generalize Theorem 1.1 of [1].

COROllary. Let $B$ be the universal enveloping algebra of a Lie algebra L. Then $J(B)=N \cdot B$, where $N$ is the nil radical of $K$.

Received by the editors November 4, 1985

1980 Mathematics Subject Classification. Primary 16A20; Secondary 17B35. 
Proof. We follow the original argument given in [1]. It is well known that in our case $N$ is the intersection of all prime ideals of $K$. If $P$ is a prime ideal of $K$, then $B / P B$ is an enveloping algebra over the domain $K / P$, and by the PoincaréBirkhoff-Witt theorem it can be filtered in such a way that $\operatorname{Gr}(B / P B)$ is a polynomial ring over $K / P$. The theorem now implies that $J(B / P B)$ is zero, and hence $J(B) \subseteq \cap P \cdot B$, where $P$ runs over all prime ideals of $K$; the latter set, by freeness of $B$ over $K$, is equal to $N \cdot B$. This, combined with the obvious inclusion $N \cdot B \subseteq J(B)$, gives the thesis.

To conclude, we will mention that the technique used above allows the rank of $L$ over $K$ to be infinite, thus removing one restriction imposed on $L$ in [1]. In addition, the characteristic of $K$ has no relevance in our reasoning at all, making the proof self-contained and simpler than in [1]. However, for this we pay the price of not being able to say anything about the primitive spectrum of $U(L)$, such information being an important by-product of the method employed in [1].

We would like to thank L. W. Small for his advice and stimulating comments, and S. Montgomery for pointing out that similar arguments are used in $[2,3]$ where she considers filtered rings whose associated graded rings have no zero divisors. Last but not least, we are grateful to R. S. Irving for his valuable and encouraging remarks.

\section{REFERENCES}

1. R. S. Irving, Primitive ideals of enveloping algebras over commutative rings, Amer. J. Math. 106 (1984), 113-135.

2. S. Montgomery, X-inner automorphisms of filtered algebras, Proc. Amer. Math. Soc. 83 (1981), 263-268.

3. X-inner automorphisms of filtered algebras. II, Proc. Amer. Math. Soc. 87 (1983), 65-74.

Department of Mathematics, University of California, Los Angeles, California 90024 